\title{
Erratum to: Diversity of methanogenic archaeal communities in Japanese paddy field ecosystem, estimated by denaturing gradient gel electrophoresis
}

\author{
Takeshi Watanabe • Makoto Kimura • Susumu Asakawa
}

Published online: 7 April 2011

(C) Springer-Verlag 2011

Erratum to: Biol Fertil Soils (2010) 46 343-353

DOI 10.1007/s00374-009-0435-2

Page 350 and Fig. 3:

"Permafrost samples only showed the existence of Methanocellales (Ganzert et al. 2007)." This sentence was incorrect. The clones estimated as Methanocellales belonged to an unknown methanogen group. The correct sentence is "Landfill samples only showed the existence of Methanocellales (Mori et al. 2003)."

We have changed the symbols in Fig. 3 according to this correction.

This correction does not influence the overall conclusions of this study.

The online version of the original article can be found at http://dx.doi. org/10.1007/s00374-009-0435-2

T. Watanabe $(\triangle) \cdot$ M. Kimura $\cdot$ S. Asakawa Laboratory of Soil Biology and Chemistry,

Graduate School of Bioagricultural Sciences, Nagoya University,

Chikusa, Nagoya 464-8601, Japan

e-mail: watanabe@agr.nagoya-u.ac.jp 

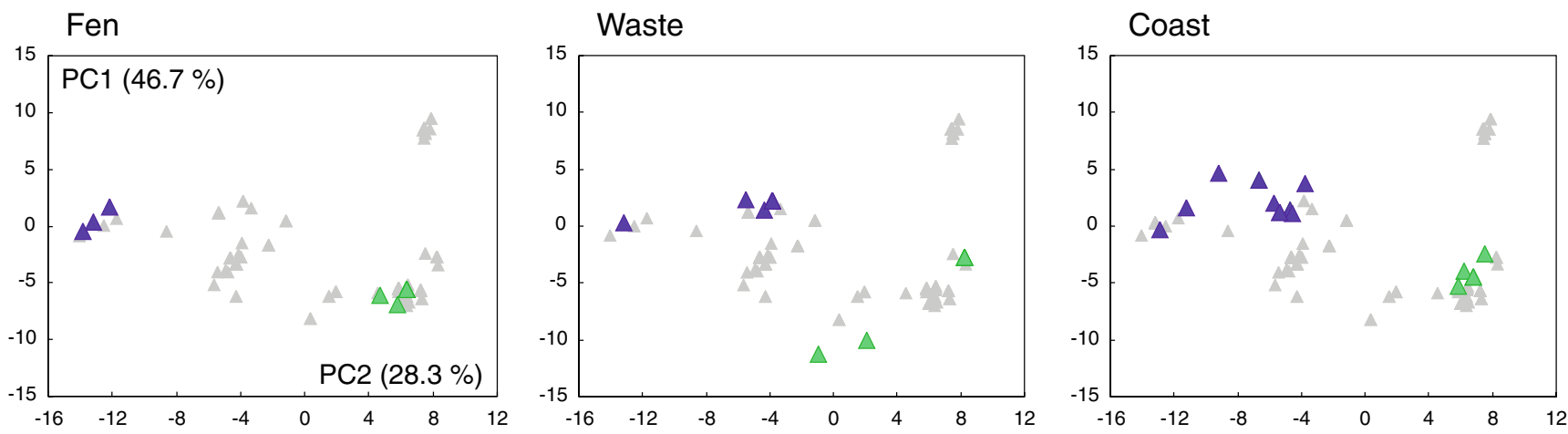

Permafrost

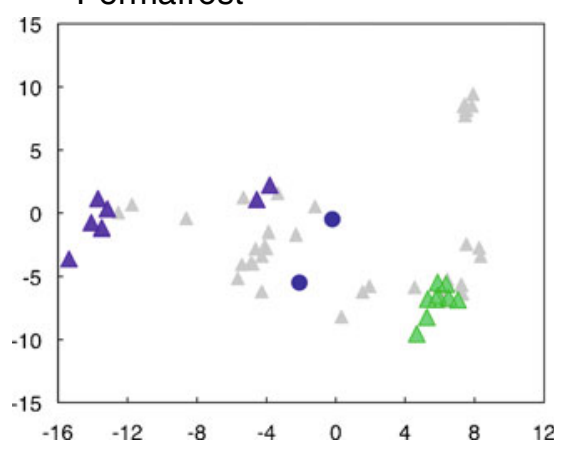

Natural gas

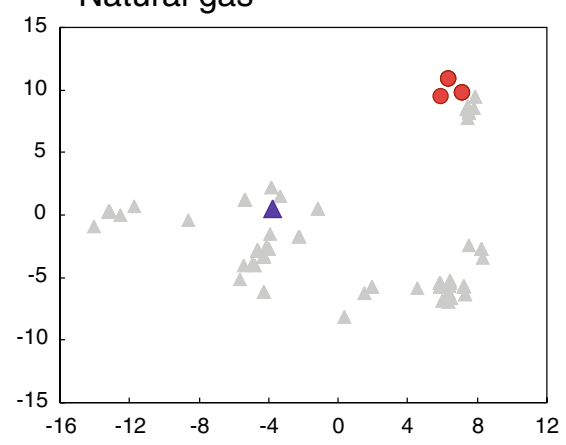

Bovine rumen

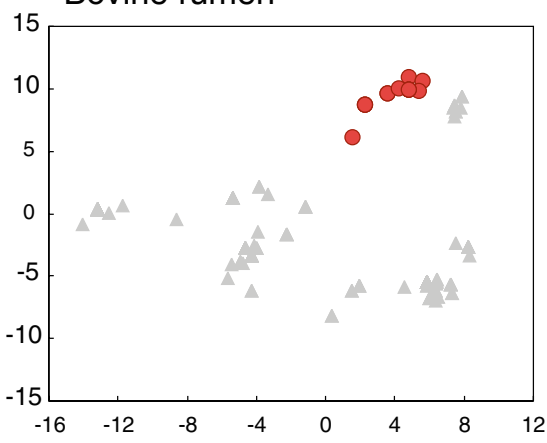

Riparian soil
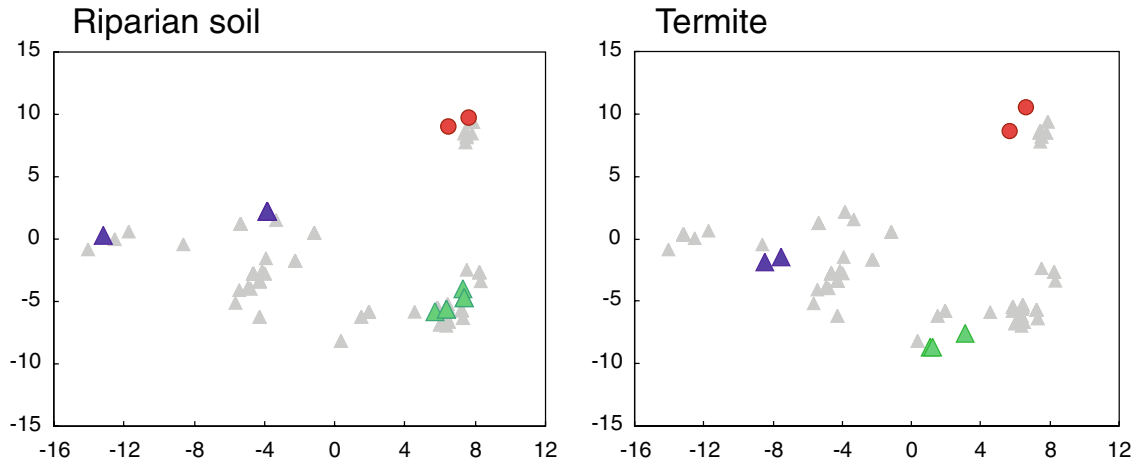

Ciliate endosymboints

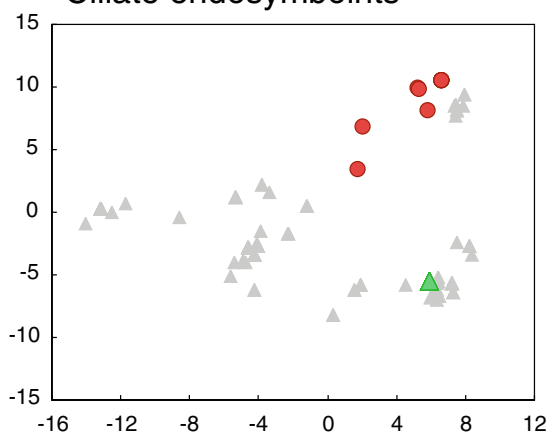

Lake sediment
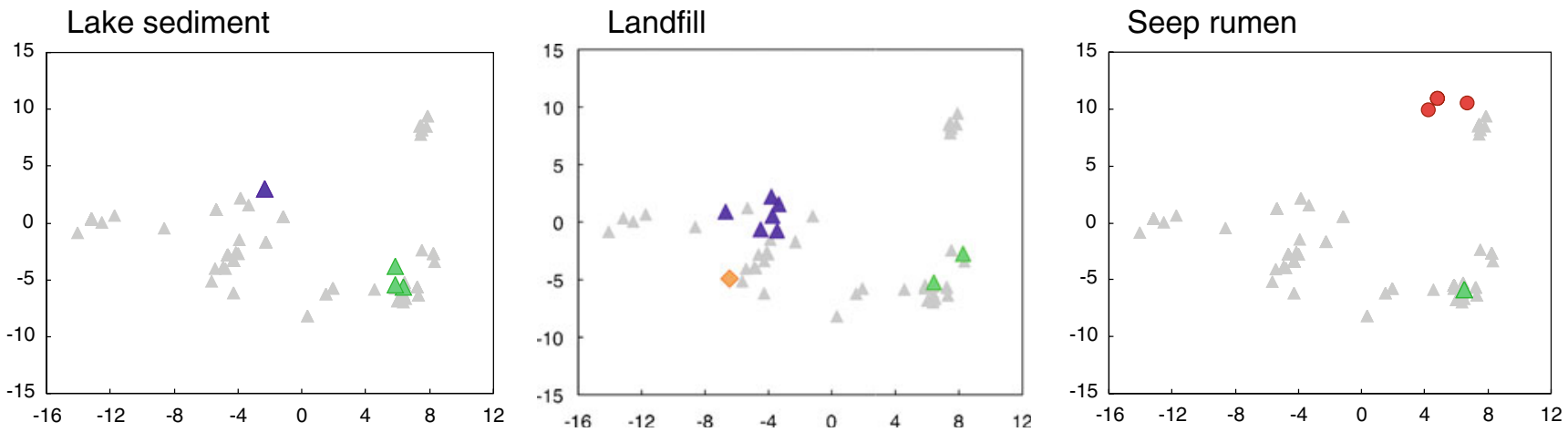

Fig. 3 Distribution maps of methanogenic archaeal clones isolated from other anaerobic environments. The principal component analysis was based on evolutionary distances of methanogenic archaeal 16S rDNA sequences (E. coli position, 358-690); the PC1 and PC2 components of the principal component analysis indicate first and second principal

components, respectively. Their respective contributing percentages are between brackets. Grey triangles indicate the clones obtained from the paddy field ecosystems; Red circle, Methanobacteriales; green triangle, Methanomicrobiales; blue triangle, Methanosarcinales; dark blue circle, unknown methanogen 